\title{
The Renormalization Flow, Spaces of Two-Dimensional Field Theories, and Connes' Geometry
}

\author{
Vipul Periwal* \\ Joseph Henry Laboratories, Princeton University, Princeton, New Jersey 08544, USA
}

\begin{abstract}
We formulate a local renormalization flow using Connes' noncommutative geometry. This formulation allows a geometric description of the renormalization flow, and an intrinsic characterization of the operator product expansion. We define spaces for string theory, in terms of a ring of correlation functions and a renormalization flow on this ring, which are the analogues of the category of Riemannian manifolds with metric for general relativity. The beta function is related to a differential form of relative entropy between two renormalization flow trajectories.
\end{abstract}

\section{The Problem}

The problem of characterizing spaces of two-dimensional field theories originates in string theory, where one would like to understand what a background independent formulation of string theory might involve. However, since the deepest aspects of string theory involve two-dimensional critical phenomena, this problem is of interest in the context of statistical mechanics as well. The "classical" equation of motion of string theory, as we presently understand it, is the condition that the nonlinear sigma model describing string propagation in a particular background be conformally invariant, which is to say, describing behaviour at a (second or higher order) phase transition. An understanding of what spaces one might embed these critical models into, might be considered analogous to understanding that solutions to Einstein's equation are objects in the category of Riemannian manifolds with metric. Furthermore, given an action, or equivalently, in terms of the Feynman path integral, a measure on the set of objects of the category, such an embedding defines the dynamics and invariance structure of the theory. The naïvest possible embedding one can envisage in string theory is that with which we are concerned in this paper, the embedding of solutions of string theory into spaces of two-dimensional field

* Address after June 10: Institute for Theoretical Physics, University of California, Santa Barbara, CA 93106 (viper (a) sbitp. ucsb. edu/viper@sbitp. bitnet), USA 
theories. In particular, we shall see indications of why critical models are "classical" solutions of string theory.

In this paper, we give a definition of spaces of two-dimensional field theories, or equivalently, spaces of two-dimensional statistical mechanics Hamiltonians, and renormalization flows on such spaces. With this data, we define "renormalization groupoids," which are possible candidates for categories of objects needed to define string theory. We have endeavoured to escape the seductions of arguments based on Feynman diagrams; our considerations are closer to real-space renormalization flow and statistical mechanics arguments. The picture presented is, to some extent, motivated by [1].

As is natural in any study of the physics of critical phenomena, the renormalization flow (RF) plays a major rôle in the following discussion. We will be concerned solely with the exact RF. The viewpoint on the action of the RF adopted in this paper is that formulated by Wilson [2, 3], Kadanoff [4], Polyakov [5], Friedan [6] and more recently, Polchinski [8], and Warr [9]. Various aspects of the RF are discussed in [10-12]. The work of Polyakov [7], Lovelace [13], and Banks and Martinec [14], is of some interest in the present context. The basic idea of renormalization is that, given a physical system, and probes available at some energy scale, the interaction of the system with these probes is described by an effective action. Local systems contain infinitely many degrees of freedom. In a nutshell, the renormalization of a quantum field theory involves constructing a sequence of cutoff approximations that describe the same low-energy physics. It is then possible to speak of the local quantum field theory, by which we mean the entire sequence of approximations. The limit of these cutoff theories, as we decrease the length scale of the cutoff, may not exist in certain cases, in the sense of having infinite coupling constants, masses and so on, but the low-energy physics we probe knows nothing of this. Throughout this paper, local will always mean local relative to scales much larger than the cutoff.

The usual picture of the RF amounts to studying the global rescaling properties of interactions. One expects that critical phenomena will involve fluctuations at all scales $[2,3,11,12]$. Hence, it is not clear that global rescalings alone are a complete framework for studying critical phenomena; by locality, there is no reason that the effective action we deduce must be derived from some bare action by global rescalings. In the operational framework of the renormalization flow, these arguments imply that we should consider a local version of the RF. When we make local changes in the scale of microscopic physics, we expect to be able to adjust the local bare couplings so as to ensure that the effective action at the observable scale remains invariant.

We may think of this, in a picturesque fashion, as follows: consider a field theory, which describes physics above a certain length scale, on a manifold, $M$, with some metric, g. The length scale is measured with respect to g. Suppose that this field theory is the effective theory that arises from some microscopic physics, described by some bare cutoff field theory, where again, the cutoff is referred to $\mathrm{g}$. The local RF amounts to studying the change in the bare cutoff field theory required to keep the effective field theory invariant, when we let the cutoff be measured with respect to a 
metric, $\mathbf{g}_{\phi}$, that is conformally equivalent to $\mathbf{g}$ :

$$
\mathbf{g}_{\phi} \equiv \exp (2 \phi) \mathbf{g} .
$$

At physical length scales, by assumption, we cannot distinguish between $\mathbf{g}$ and $\mathbf{g}_{\phi}$, by measuring any observable invariant charge [16], e.g., dimensionless combinations of a physical length and curvature.

In string theory, we are interested in amplitudes, $G_{I}$, describing the interactions of excitations of the string background. These are obtained from correlation functions of vertex operators on the string worldsheet, by integrating over the positions of the vertex operators on the worldsheet. These integrated amplitudes are the equivalent of low-energy physics in the above discussion; "low-energy," in the context of the worldsheet, refers to the zero eigenvalue of the Laplacian on the worldsheet, which corresponds to functions constant on the worldsheet (assuming that the worldsheet is compact and without boundary). These amplitudes are functions that are constant along trajectories of the RF; they are the invariant charges of the RF [16]. Their definition involves understanding the local field theory on the string worldsheet: if $H$ stands for a certain two-dimensional statistical mechanics system, defined with respect to some metric $\gamma$ and cutoff $\varepsilon$, upon changing the metric to $\exp (2 \phi) \gamma \equiv \gamma^{\prime}$, we change the "couplings" of the system to $H^{\prime}$ such that integrated amplitudes are constant,

$$
G_{I}(H, \gamma)=G_{I}\left(H^{\prime}, \gamma^{\prime}\right)
$$

This should be compared to the usual procedure in quantum field theory (see, e.g., [16]) where $S$-matrix elements and poles in physical propagators are examples of invariant charges. In that situation, these physical parameters satisfy homogeneous renormalization flow equations, albeit for a global version of the RF. We shall be interested in a local version of the RF, which we have seen may be geometrically formulated in terms of conformal changes of the metric. The constant eigenfunctions of the Laplacian are invariant under arbitrary changes of the metric. Hence, the local $\mathrm{RF}$ enables us to go from a pair $(H, \gamma)$ (in the notation of the pevious paragraph) to $\left(H^{\prime}, \gamma^{\prime}\right)$ satisfying (1.1), where $\gamma^{\prime}$ is any metric obtained from $\gamma$ by conformal diffeomorphisms and Weyl rescalings. In $t$ wo dimensions, conformal equivalence is a particularly simple restriction on the geometry on which the system lives. Conformal equivalence is, of course, also of geometric interest in higher dimensions [17].

We return now to the motivation mentioned in the first paragraph of this section. We expect that two-dimensional statistical mechanical systems for a given set of observables (up to an intrinsic equivalence structure, to which we return in the following) provide a suitable configuration space for string theory. Therefore, motivated by the Feynman path integral, we want to consider an integral of the form

$$
\int_{\hat{A}} \mathscr{D} \mathscr{M}
$$

where $\hat{A}$ is "a space of two-dimensional Euclidean field theories," and $\mathscr{D} \mathscr{M}$ is some measure on $\hat{A}$, which we may think of as being of the form $\mathscr{D} \mathscr{M} \equiv \mathscr{D} H \exp (-S)$. For orientation, we compare (1.2) to its analogue in the theory of gauge fields, where we 
consider

$$
\int_{\mathscr{G}(P)} \mathscr{D} A \exp \left(-\|F(A)\|^{2}\right)
$$

here, $\mathscr{C}(P)$ is the space of connections on a principal $G$-bundle, $P, G$ is a compact simple Lie group, and $\mathscr{D} A \exp \left(-\|F(A)\|^{2}\right)$ is the Yang-Mills measure. Expression (1.3) is ill-defined as it stands, because the Yang-Mills measure is invariant under automorphisms of $P$. We expect the same situation to hold for (1.2), though we are somewhat hampered by the fact that we do not know what $\hat{A}$ is, we do not know what $\mathscr{D} \mathscr{M}$ is, nor do we know the analogues of the automorphisms of $P$ for (1.2).

We consider the physics that (1.2) models, in order to motivate our answer to the last two of these problems. The first problem will occupy us for a large part of this paper. When we study strings in some background, i.e., study the correlations of vertex operators, $\mathcal{O}_{i}$, weighted by some Hamiltonian, $H_{0}$, the Polyakov integral for the correlation of $\mathcal{O}_{i}$ is written as

$$
\left\langle\mathcal{O}_{1} \mathcal{O}_{2} \ldots\right\rangle_{H_{0}} \equiv \sum_{k=0}^{\infty} g^{k} \int \mathscr{D} \gamma \int \mathscr{D} X \exp \left(-H_{0}\left[\gamma, X, \partial^{(n)} X\right]\right) \mathcal{O}_{1} \mathcal{O}_{2} \ldots,
$$

where $\mathscr{D} \gamma$ is a measure for integrating over all metrics on a two-dimensional surface of genus $k, g$ is an additional parameter (the coupling constant), and $\mathscr{D} X \exp \left(-H_{0}\left[\gamma, X, \partial^{(n)} X\right]\right)$ is a measure for an integral over configurations of the string worldsheet in the background corresponding to the local (i.e., involving only a finite number of derivatives of $X$ ) Hamiltonian, $H_{0}$. We find that those $H_{0}$ for which the integral over metrics decouples (up to a finite-dimensional integral over moduli) are simple to treat in this picture; these are the conformally invariant theories. Of course, only for some $\mathcal{O}_{i}$, the conformally invariant operators, does the integral over metrics in (1.4) decouple.

These conformally invariant correlations describe certain fluctuations, $h$, about $H_{0}$, in a manner that we shall indicate presently. Here, we note that it is (believed that it should be) possible to find $S_{\mathrm{ft}}\left[g, H_{0}, h\right]$ such that the field theory defined by the Feynman path integral

$$
\int \mathscr{D} h \exp \left(-S_{\mathrm{ft}}\left[g, H_{0}, h\right]\right)
$$

is a generating functional which reproduces the conformally invariant correlations of the Polyakov approach. The conformal invariance of the statistical model described by $H_{0}$ shows up as gauge invariance in the field theory described by (1.5). In formulating (1.5), we lose some of the invariances of (1.4); we have to make a choice of conformal frame in going from (1.4) to (1.5). Of course, (1.5) also defines correlations of operators that are not invariant under conformal transformations, but we have no means of connecting these to the Polyakov picture, since they depend on the conformal frame that defines (1.5). It is, in a formal sense, posible to eliminate the choices involved in setting up (1.5): all we need do is to average over them, which would bring us to

$$
\int \mathscr{D} \gamma \mathscr{D} h \exp \left(-S_{\mathrm{ft}}\left[g, H_{0}, \gamma, h\right]\right) / \int \mathscr{D} \gamma .
$$

Expression (1.6) looks formally similar to (1.2), if we consider $\gamma$ and $h$ as jointly 
describing fluctuations about the Hamiltonian, $H_{0}$. Of course, this is overkill, since we must now attempt to make sense of (1.6); neither does this lead to any insights as to why $H_{0}$ was a "good" Hamiltonian from the point of view of (1.4).

While (1.6) superficially resembles (1.2), our dissatisfaction with (1.6), or for that matter with (1.5), leads us to attempt to make sense of (1.2) without going through the path indicated in the previous paragraph. Therefore, keeping the local twodimensional aspects of the worldsheet in mind, we consider now a different "decomposition" of $\hat{A}$. Spaces of Hamiltonians only make sense if we consider them as spaces of cutoff Hamiltonians. Since a cutoff presupposes a metric, when we speak of a cutoff Hamiltonian, $H$, in the following, we shall implicitly assume that we are given a pair $(H, \gamma)$, as in our previous discussion of the local RF. In line with our remarks about invariant charges of the RF, assume that $\mathscr{D} \mathscr{M}$ is such that $S[H]=S\left[H_{\text {eff }}\right]$, if $H$ lies on the RF trajectory labelled $H_{\text {eff }}$. We may then use the natural action of the RF on spaces of cutoff Hamiltonians as motivation for writing the measure in (1.2) as

$$
\mathscr{D} \mathscr{M} \equiv \mathscr{D} H \exp (-S[H]) \equiv \mathscr{D} H_{\text {eff }} \exp \left(-S\left[H_{\text {eff }}\right]\right) \mathscr{D} R\left(H_{\text {eff }}\right),
$$

where $\mathscr{D} H_{\text {eff }}$ denotes a measure on "a space of renormalization flow trajectories," and $\mathscr{D} R\left(H_{\text {eff }}\right)$ denotes a suitably normalized measure along the local RF trajectory that defines the effective Hamiltonian, $H_{\text {eff }}$. (A natural candidate for $\mathscr{D} H$ is provided by the GNS metric, which we discuss below.)

One of the questions we address is the possibility of making sense of such a decomposition, (1.7). Of course, this is equivalent to the existence of the local renormalization flow, so our effort is mainly to cast the physics of the RF in a precise and suitable framework. We note that the decomposition in (1.7) is somewhat in the spirit of Faddeev-Popov. This is our basis for understanding the gauge symmetry of string theory, the equivalence under the local renormalization flow. We conjecture that the term involving $S$ in (1.7) is actually absent. This provides a simple explanation for why critical points of the RF are "classical" solutions of string theory. We expect that "many" (or very few) RF trajectories pass through fixed points of the RF, depending on the attractive (or repulsive) character of the fixed point. Heuristically, since integrated amplitudes are invariant charges for the local $\mathrm{RF}$, consider a (local) parametrization of the space of trajectories in terms of some physical quantities, say, values of certain amplitudes, $G_{I}$. The number density of RF trajectories, $n\left(G_{I}\right)$, with values for these amplitudes close to $G_{I}$, is the integrand of $\mathscr{D} G_{I}$ in these coordinates. This could be considered as the Jacobian for moving from intrinsic to "physical" coordinates. In other words, if we wished to specify the measure, $\mathscr{D} \mathscr{M}$ in $(1.2)$ as $\mathscr{D} G_{I} \exp \left(-S_{G_{I}}\right)$, we would define

$$
S_{G_{I}} \propto-\ln n\left(G_{I}\right)
$$

as the "action" for string theory. Attracting critical trajectories are those points where $n\left(G_{I}\right)$ is locally extremal.

Implicit in the conjecture formulated in the previous paragraph is the idea that the entropy (which should not be confused with the relative differential entropy we introduce in Sect. 5) of the local renormalization flow governs dynamics. We consider the fact that Planck's constant and temperature play analogous rôles in 
Feynman path integrals, the former appearing in quantum mechanics and the latter in statistical mechanics. In this light, the absence of Planck's constant is equivalent to the statement that string theory is defined at infinite temperature. The (attracting) critical trajectories are dominant in (1.7) because they correspond to the effective Hamiltonians with a locally extremal number density of RF trajectories - this is the sense in which they are the "classical" solutions of string theory. Following standard procedures in field theory, we would like to use this to define an effective action.

We have made no mention of moduli in the above discussion. We could restrict our discussion to two-dimensional field theories defined on a given compact twodimensional surface. However, we wish to provide a substitute for (1.4) or (1.5). The main thrust of our approach is to consider cutoff two-dimensional field theories in a unified manner, without any attempt to extract the dependence on the geometry. Therefore, we must consider these field theories or statistical mechanical systems to be defined on a suitably flexible set of two-dimensional manifolds. We will say nothing about moduli in the main body of this paper, leaving this to future work. The local renormalization flow does not relate metrics belonging to different conformal equivalence classes, which implies that, as far as the space of local RF trajectories is concerned, distinct points in moduli space give rise to distinct local RF trajectories. We could, if we so wished, attempt to decompose the measure on the space of trajectories as

$$
\mathscr{D} H_{\text {eff }} \equiv d m \mathscr{D} H_{\text {eff }}^{m} \text {; }
$$

we do not see any physical reason for actually doing so. In the present work, we restrict ourselves to two-dimensional field theories defined on a compact topological surface of finite genus.

We turn now to a discussion of how to describe spaces of Hamiltonians. To be able to speak in a precise manner about "spaces of statistical mechanics Hamiltonians," or of the RF on such spaces, we need some new tools. In particular, we shall be interested in considering the foliation (defined in the appendix) of such spaces by the local RF. The leaves of this foliation are the trajectors of the RF, or, in the language of the previous paragraph, the effective Hamiltonians of the given space of cutoff Hamiltonians. Since our interest is in critical phenomena, especially with the motivation given above, we are interested in foliations that degenerate, which means that the situations we need to deal with are singular in terms of usual differential geometry or topology.

In the past few years, A. Connes has sketched the outlines of a powerful set of geometric constructs in operator theory which are applicable to many situations where the usual tools of topology and geometry make no sense. The definition of renormalization groupoids that we formulate, in terms of Connes' constructs, is natural. Given the singular nature of spaces of leaves of foliations, standard differential geometry does not suffice to understand measures on such spaces. An understanding of the generating functional of transverse measures on renormalization groupoids needs Connes' non-comutative integration theory [18].

We use the language of statistical mechanics [20]. The (two-dimensional) nonlinear sigma model path integral is, for the purposes of string theory, regarded as a sum over spacetime trajectories of a string, hence the worldseet interpretation. Here, 
we regard it as a statistical trace, weighted by some Hamiltonian. In particular, regard the path integral as a statistical trace,

$$
\int D X \exp \left(-S_{\sigma}\right) \equiv \operatorname{Tr}\left\langle X\left|\exp \left(-H_{\sigma}\right)\right| Y\right\rangle,
$$

where $S_{\sigma} \equiv H_{\sigma}$ is regarded as a statistical Hamiltonian on the right-hand side. (This has nothing to do with the path integral, with boundary conditions, as a transition amplitude in quantum mechanics.) $S_{\sigma}$ may be thought of as the integral of a local function of $X$ and derivatives of $X$, up to some finite order. The path integral is understood here as being done with some set of boundary conditions suited to statistical mechanics, and we have not performed any integral over metrics, as we would if we were following Polyakov's approach. Therefore, both sides of the equation depend implicitly on a metric on some two-dimensional manifold. In this formulation, one has a better picture of what is involved when one attempts to formulate what a space of two-dimensional field theories ought to be, i.e., we may think of spaces of states of some statistical mechanical system, as we now describe.

In statistical mechanics, the notion of a Boltzmann average has been codified into the definition of a state on a $C^{*}$ algebra. More precisely, we consider a space of observables, which is an abstract algebra, $A$. These need not strictly be "physically" observable fields. A state on $A$ is a linear functional on $A$, such that if $\rho: A \rightarrow \mathbf{C}$ is a state, then

$$
\rho\left(a^{*} a\right) \geqq 0, \quad \forall a \in A .
$$

This last condition is the positivity condition, and we do not expect it to hold in theories with gauge symmetries, except in a subspace. (The usual definition in this context is somewhat more involved.)

Denote the space of states on $A$ by $\hat{A}$. There is much more structure that can be (naïvely) attached to such a space. Recall the Gel'fand-Naimark-Segal(GNS) construction. This allows us to canonically associate a Hilbert space $\mathscr{H}_{\rho}$ to given state $\rho$, by noting that, for $a, b \in A$,

$$
\langle a b\rangle \equiv \rho\left(a^{*} b\right)
$$

defines an inner product, provided that we factor out the subspace generated by $a \in A: \rho\left(a^{*} a\right)=0$. Thus, one imagines a vector bundle over the space of states, which is easily seen, from (1.8), to contain the information in correlation functions. The GNS construction also supplies a $*$ homomorphism

$$
A \rightarrow \mathscr{B}\left(\mathscr{H}_{\rho}\right),
$$

into the bounded operators on $\mathscr{H}_{\rho}$. We may therefore consider a bundle of operators over $\hat{A}$,

$$
\mathscr{B}\left(\mathscr{H}_{\rho}\right) \rightarrow \begin{aligned}
& \mathscr{B} \\
& \downarrow \\
& \widehat{A} .
\end{aligned}
$$

Roughly speaking, we shall describe $\hat{A}$ by means of the "matrix elements" of sections of $\mathscr{B}$ acting on the space of sections of $\mathscr{H}$. We note that $\hat{A}$ is not the space of all states on $A$ in our definition. The GNS metric, defined by (1.8), is the natural metric on $\hat{A}$ 
because the vanishing of its curvature is exactly the strong associativity condition of the operator product expansion [22].

This paper defines spaces of two-dimensional statistical mechanical systems, in terms of spaces of correlation functions and the action of the renormalization flow on such spaces, as understood in terms of Connes' constructs. The motivation for our constructions is provided by (1.7). The idea is simple: we make the (given) space of correlation functions into a ring in a suitable way, heuristically motivated in the above discussion. We define the space of states of this ring, via the endomorphisms of the module corresponding to the ring, as a certain subset in the automorphisms of the module. A renormalization groupoid is then defined so as to describe this space of states modulo an equivalence relation, two states being equivalent if they lie on the same renormalization flow trajectory.

This paper is organized as follows:

- In Sect. 2, we sketch some constructions due to Connes.

- In Sect. 3, we formulate the problem in a suitable language for the use of Connes' tools.

- In Sect. 4, we formulate the renormalization flow in terms of the constructions of Sect. 3.

- In Sect. 5, we discuss various physical consequences of our formulation. We relate aspects of the beta function to a relative differential entropy between two renormalization flow trajectories.

- In Sect. 6, we make some concluding remarks.

An appendix contains a glossary of mathematical terminology.

\section{Connes' Geometry}

Connes' geometry is based on a simple idea: study a space through an algebra associated to it. A locally compact topological space, $X$, can be described completely in terms of the algebra of continuous functions $\mathscr{C}(X)$ on it. The Serre-Swan theorem [26] tells us that finite-dimensional vector bundles over a manifold, $X$, are equivalent (in the sense of $\Gamma$, as defined below, being an equivalence of these categories) to finitely-generated projective modules over $\mathscr{C}(X)$, i.e., a vector bundle, $V$, can be described by its sheaf of sections, $\Gamma(V)$, with the natural action of $\mathscr{C}(X)$ by multiplication:

$$
\begin{aligned}
f: \Gamma(V) & \rightarrow \Gamma(V) & \forall f \in \mathscr{C}(X), \\
s & \mapsto f s, & \forall s \in \Gamma(V) .
\end{aligned}
$$

The tangent bundle of a manifold can also be replaced by an algebraic construct, in a very natural way. Let $R \equiv \mathscr{C}(X)$ in the following. A derivation of a ring, $R$, is an element $t \in \operatorname{Der}(R)$ such that

$$
t(f g)=f t(g)+t(f) g
$$

The derivations from a Lie algebra, $\operatorname{Der}(R)$. In this setting, the tangent space of $X$ is understood through $\operatorname{Der}(R)$. This is natural, because if one studies a space by means of the functions on it (here the ring $R$ ) then the action of the tangent vectors is 
through the sections of the tangent bundle acting on the elements of $R$ as differential operators:

$$
\begin{gathered}
\operatorname{Der}(R) \times \mathscr{C}(X) \rightarrow \mathscr{C}(X) \\
\left(v \equiv \sum v_{i} \frac{\partial}{\partial x_{i}}, f\right) \mapsto v(f) \equiv \sum v_{i} \frac{\partial}{\partial x_{i}} f .
\end{gathered}
$$

If a Lie algebra acts on $X$, there are elements of $\operatorname{Der}(R)$ that represent the Lie algebra. A foliation of a manifold is defined as an integrable sub-bundle of the tangent bundle. An elementary theorem of Frobenius relates this to an equivalent description of a foliation: a sub-bundle is integrable, iff the sections of the subbundle form a Lie subalgebra of $\operatorname{Der}(R)$. Therefore, the spaces of leaves of foliations or quotient spaces of Lie algebra actions can be described in algebraic terms.

The importance of these observations lies in the fact that these algebraic descriptions continue to make sense even when there is no sensible topological space underlying the algebra. Furthermore, the geometry of these algebras can be naturally extended to non-commutative algebras, which turn out, in fact, to be essential to the study of the $K$-theory of even the commutative algebras [25]. For example, non-commutative algebras enter naturally in the proof of Bott periodicity [26].

We now sketch some of Connes' constructs [23-25] that we shall use later to define the action of the renormalization flow on the space of states corresponding to a given algebra of observables. Let $R$ be a ring, and let $S$ be a module over $R$. We shall be interested in the case that a Lie algebra, $l$, acts on $R$. As described above, this means that we have a Lie algebra homomorphism $l \rightarrow \operatorname{Der}(R)$. A connection, $\delta$, in this context, is an element of $\operatorname{Hom}\left(R, R \otimes l^{*}\right.$ ), which we may write as (for $f, g \in R$, and $v \in l)$

$$
\begin{aligned}
\delta: R & \rightarrow R \otimes l^{*}, \\
f & \mapsto \delta f \in R \otimes l^{*} .
\end{aligned}
$$

This connection satisfies the Liebniz rule:

$$
\delta(f g)=f \delta g+\delta f g
$$

and, of course, $\delta f: v \mapsto \delta_{v} f \in R$. Curvature is defined as usual:

$$
\theta(v, w)=\delta_{v} \delta_{w}-\delta_{w} \delta_{v}-\delta_{[v, w]}
$$

and is an element of End $(R) \otimes \Lambda^{2} l^{*}$. When the connection has curvature, it does not induce a homomorphism from $l \rightarrow \operatorname{Der}(R)$. Here, by assumption, the connection is flat, $\theta=0$.

Now, given a finitely-generated projective module, $S$, over $R$, it is possible to define a connection on $S$, i.e., a map $\nabla: S \rightarrow S \otimes l^{*}$, which "extends" the connection on $R$, such that for $r \in R, \chi \in S$, one has, regarding $S$ as a left $R$-module,

$$
\nabla(r \chi)=r \nabla \chi+\delta r \chi
$$

Connes defines this lift to preserve a hermitian metric on $S$, i.e., a hermitian form $\langle$,$\rangle :$ $S \times S \rightarrow R$. One may attempt to find a lift of the action of $l$ (via the connection, which 
on $R$ is flat by assumption) to $S$, so as to satisfy the above requirement. We define the curvature of this lift, $\Theta \in \operatorname{End}_{R}(S) \otimes \Lambda^{2} l^{*}$, as in (2.2), and again a representation of the Lie algebra, extending the assumed representation on $R$ is obtained, if the connection, $\nabla$, is flat.

Our motivating idea here is that of a flat connection defining a foliation of $\hat{A}$ as endomorphisms of $S$. In our case, $S$ will be a module over a ring, Fock $R$, of functions on some surface. $S$ is a ring of correlation functions, in terms of which we shall define the space of states on some algebra of observables. The Lie algebra we are interested in is the Lie algebra of conformal diffeomorphisms and Weyl rescalings of the metric on the surface, which acts on $R$, and, with some choice of lift, on $S$.

We now come to a construct that is central to our understanding of the decomposition of the measure in (1.7). This is the notion of a holonomy groupoid, due to Winkelnkemper (as described in [24]). We refer to [24] for a complete description. Given a manifold, $V$, with a foliation, $F$, we wish to construct an object that will describe the space of leaves, $V / F$, i.e., a partition of the manifold into equivalence classes, with two points in $V$ belonging to the same equivalence class iff they lie on the same leaf of the foliation, $F$. Consider the set $\hat{G} \equiv V \times V \times P V$, where $P V$ denotes the set of maps from $I \equiv[0,1] \rightarrow V$. Define $G_{0} \subset \hat{G}$, as consisting of the elements in $\hat{G}$ of the form $(x, y, p)$ with $p(0)=x, p(1)=y$, and

$$
\frac{d p}{d t}(t) \in F_{p(t)}, \quad \forall t \in I ;
$$

these are paths lying on the leaves of the foliation.

There is a natural map $q: T V \rightarrow T V / Q$, at each point of $V$. Bott [27] defined a connection for transport in the normal bundle tangent to each leaf of the foliation via:

$$
\nabla_{X}^{B} S \equiv q([X, Y]),
$$

where $X \in F, s$ is a section of $Q$ and $Y \in T V: q(Y)=s$. Since $F$ is integrable, and, by definition, in the kernel of $q$, we see, using the theorem of Frobenius, that this is welldefined on $Q$. Furthermore, the Jacobi identity implies that this is a flat connection, called the Bott connection. This allows us to define a holonomy map, i.e., a map $h$ defined on the paths $p$ of elements $(x, y, p) \in G_{0}, h(p) \in Q_{y} \otimes\left(Q_{x}\right)^{*}$. By the flatness of the connection, the only closed paths with non-trivial holonomy are non-contractible. This motivates the following definition: the holonomy groupoid of $(V, F)$ is defined to be

$$
G \equiv G_{0} / \sim, \quad \text { where } \quad(x, y, p) \sim\left(x, y, p^{\prime}\right) \Leftrightarrow h(p)=h\left(p^{\prime}\right) .
$$

It is also of interest to compute the holonomy of the GNS metric. $G$ has a natural composition structure, arising from the composition of paths, which makes it a groupoid.

This is the simple and intuitive basis for the construction of an algebra $C^{*}(V, F)$ to attach to $V / F$. We shall not describe this algebra precisely here, since we make no use of it in the present treatment. Measures on the space of RF trajectories are understood entirely in terms of this algebra. The physics of string theory is contained in these measures. Here, we note that intuitively, one may think of $C^{*}(V, F)$ as 
"matrices" of the form $k_{x, y}$ where the "indices" $x, y$ lie on the same leaf of $(V, F)$, with a product appropriate to a convolution algebra on the holonomy groupoid. $C^{*}(V, F)$ is local, i.e., given an open $V^{\prime} \subset V$, and $F^{\prime}=\left.F\right|_{V^{\prime}}$, there is a *-isometric homomorphism $C^{*}\left(V^{\prime}, F^{\prime}\right) \rightarrow C^{*}(V, F)[24]$.

\section{The Formalism}

Our formalism, in accordance with the ideas described in the previous section, is defined in terms of function rings. Therefore, our considerations are both coordinate-independent and independent of the topology of the surface. We assume that we are given a real, $C^{\infty}$ two-dimensional manifold, $O$, the space on which our statistical system lives, or the string worldsheet. Pick a ring of functions over $O$, call it $R$. We assume that the elements of $R$ are infinitely differentiable; take $R \equiv C^{\infty}(O)$, for example. The infinite differentiability we have assumed is not a restriction [23]. In the present context, because we deal with cutoff systems on compact domains, we will have physical reasons for assuming that everything we consider is smooth. This is related to the fact that the non-analyticities that characterize phase transitions only arise in the thermodynamic limit.

$A$ is a set of observables, which we think of as classical fields on $O$. In their rôle as classical fields, elements of $A$ can be defined at each point, and multiplied as well. However, we shall have no use for this algebraic structure, since it makes little sense quantum mechanically. We base our arguments here on the assumption that we are given some basic set of "classical fields", $\Phi_{i}(\xi), \xi \in O$, which include an identity element $e: e \Phi_{i}(\xi)=\Phi_{i}(\xi)$. (See [22] for examples of this.) The elements of $A$ are local functions (i.e., with only a finite number of derivatives) of these fields, that have been smeared (in the sense of the Wightman definition of fields as operator-valued distributions [29]). They may be thought of as linear combinations of elements of the form (for $f \in R$ )

$$
\phi(f) \equiv \int_{O} \phi(\xi) f(\xi)
$$

where $\phi$ is some local function $\phi=\phi(\Phi)$, of our basic fields. The action of $R$ on this space is simply as

$$
[g] \phi(f) \equiv \phi(g f)
$$

This arises from the natural homomorphism,

$$
\begin{aligned}
{[\quad]: R } & \rightarrow \operatorname{End}(A) \\
f & \mapsto[f],
\end{aligned}
$$

so $A$ is an $R$-module. (In the following, we will treat the square brackets as understood.)

A maximal ideal of $R$ is a subring of $R$ that contains functions vanishing at some point, $p \in O$. We assume that the module, $A$, is a local module with respect to $R$, i.e., for any two (distinct) maximal ideals, $m_{i}, i=1,2$, of $R$,

$$
A / m_{1} A \cap A / m_{2} A=0 .
$$


The intuition for this is as follows: when we pick two distinct maximal ideals, we are looking at functions that vanish at two separate points, and the factor modules $A / m_{i} A$ contain only those operators that are supported at exactly those two distinct points. Therefore, this requirement, (3.1), is a physical one, we are simply assuming that "field" degrees of freedom at two distinct "points" are independent.

Assume that there are operators in $A$ that represent intrinsic information about the metric on $O$, such as covariant derivatives and curvatures. In particular, this implies that we study statistical models including dependence on geometry. Part of the information in the state is the choice of a metric on $O$. Elements of $A$ do not have any connection to a given metric on $O$, until they are represented via a state. Furthermore, one can, of course, define derivatives of operators (schematically) through the usual definitions:

$$
\phi(\partial f) \equiv \int_{O} \partial f \phi=-\int_{O} f \partial \phi=-\partial \phi(f)
$$

(We ignore boundary terms in the present work. These are important [28].) The identity element, $e$, allows us to identify an $R$-submodule of $A$, which is denoted $[R]$ $e$ and consists of elements obtained as follows:

$$
R \ni f \mapsto \int_{O} f e \in A
$$

so that we write

$$
A=[R] e \oplus A_{0} .
$$

A final point is that in this whole scheme, it is necessary to consider, not just $R$, but

$$
\text { Fock } R \equiv \bigoplus_{n=0}^{\infty} R^{\otimes n}, \quad \text { and } \quad \text { Fock } A \equiv \bigoplus_{n=0}^{\infty} A^{\otimes n} \text {. }
$$

We define

$$
R^{\otimes 0} \equiv \mathbf{R}, \quad \text { and } \quad A^{\otimes 0} \equiv \mathbf{R} .
$$

We consider Fock $A$ as a Fock $R$-module in the obvious way.

On a space of statistical mechanics states (or equivalently, of Euclidean field theories) there is a natural space of "functions"-the correlation functions. An advantage of describing spaces of states in terms of correlation functions is that we automatically work with constructs that are independent of the method of regularization, provided we use physical regulators. Dimensional regularization, for example, is excluded since its action on correlation functions is transcendental, because it takes us "outside the ring." We will describe (and topologize) the space of states, $\hat{A}$, solely by means of correlation functions. Therefore, two states that arise from different regulators, e.g., a lattice regulator and a momentum-space cutoff, but have the same value for all correlation functions, are identified. Clearly, we need to say more before it makes sense to compare the values of correlation functions for two different regulators, but we certainly expect a map between the observables in any two schemes. This is another reason why transcendental regulators cannot be used in our approach. We indicate a natural equivalence between different physical regulators in Sect 5. 
Consider the algebra defined by correlation functions, considered as "functions" on the space of states. To be precise, $\forall a \in$ Fock $A$, define a function, $s_{a}$, on the space of states in the obvious fashion, i.e., for $\rho \in \hat{A}$,

$$
s_{a}(\rho) \equiv \rho(a) .
$$

Explicitly, if $a \equiv a_{1} \otimes a_{2} \ldots a_{n} \in A^{\otimes n}$, then

$$
s_{a}(\rho) \equiv \rho\left(a_{1} a_{2} \ldots a_{n}\right) .
$$

Call this set of functions, $S$, and note that $S=\oplus_{n=0}^{\infty} S_{n}$, with the natural grading induced from the Fock structure of Fock $A$. One doesn't a priori know how to multiply elements of $S$; there is just the product induced from the product in Fock $A$,

$$
\left(s_{a} \otimes s_{b}\right)(\rho) \equiv \rho(a b) \equiv s_{a \otimes b}(\rho),
$$

which makes $S$ into a ring. We think of $S$ as the "structure ring" of $\hat{A}$. However, this is obviously a very non-trivial algebraic structure, so it is necessary to exercise a great deal of care. In particular, we will describe $\hat{A}$ solely in terms of $S$, so we regard $S$ as the fundamental object.

$S$ is naturally a Fock $R$-module:

$$
\begin{gathered}
R^{\otimes n} \ni f_{1} \otimes f_{2} \ldots \otimes f_{n}: S_{n} \rightarrow S_{n} \\
S_{\phi_{1} \otimes \cdots \otimes \phi_{n}} \mapsto S_{\left(f_{1} \phi_{1}\right) \otimes \cdots \otimes\left(f_{n} \phi_{n}\right)},
\end{gathered}
$$

where $f_{i} \in R, \phi_{i} \in S$, and $s_{\phi_{1} \otimes \cdots \otimes \phi_{n}}\left(\in S_{n}\right)$ is an $n$-point correlation function. (Here we have defined the map on generators of $S_{n}$, the extension to arbitrary elements is via linearity.) For an element $s_{\phi}$ in $S_{n}$, we can define the support of $s_{\phi}$, which we denote $\operatorname{Supp}\left(s_{\phi}\right) \subset O^{n}$, as the closure of the set of points over which $\phi$ is smeared.

We draw attention, in particular, to $S_{0}$, which is a module over $\mathbf{R} \equiv R^{\otimes 0}$, generated by a section we denote $Z$, so $S_{0}=\mathbf{R} Z$. We call $Z$ the partition function. In view of our previous definitions, we note that this is justified because

$$
Z(\rho) \equiv \rho(1), \quad \rho \in \hat{A} \text {. }
$$

$Z$ plays the rôle of the identity in the multiplication in $S$, as defined in (3.5).

We wish to study the space, $\hat{A}$. It is clear that the $\operatorname{ring} S$ contains all the physical information that we need to describe $\hat{A}$. For example, suppose we pick a point in $\hat{A}$. We think of this point, intuitively, as having a Hamiltonian associated to it, which we denote $H$, and we write the associated correlation functions as $s_{a}\left(\rho_{H}\right) \equiv \rho_{H}(a)$, for $a \in$ Fock $A$. Then, the correlation functions of a perturbation of this Hamiltonian, denoted $\delta H$, are naïvely recovered from

$$
\rho_{H+\delta H}(a)=\sum_{n=0}^{\infty} \frac{(-)^{n}}{n !}\left[\left(s_{\delta H}\right)^{\otimes n} \otimes S_{a}\right]\left(\rho_{H}\right) .
$$

We have assumed that $s_{\delta H} \in S$; this is part of the more basic assumption that the states are describable from within the algebra, $A$. This is not a restrictive assumption, since we allow ourselves arbitrary (finite) linear combinations of elements in $A$, and deal with a compact manifold, $O$. The sum converges because $O$ has finite volume and every state is assumed to be provided with a cutoff. (This description of $\hat{A}$ is 
intended only in a local sense; we give a precise definition in Sect. 5.) When we study the general case of a disjoint union of surfaces, as sketched in Sect. 1, we will need to work somewhat harder.

We are now in a position to define the renormalization flow on $\hat{A}$, through its action on $S$. Cutoff dependence is a generic feature of the path integrals (equivalently, traces) that define the physics of Euclidean quantum fields (statistical mechanics systems, in the thermodynamic limit). If we exclude exactly critical and ultralocal models, the action of Weyl rescalings of the metric and conformal diffeomorphisms on correlation functions is quite nontrivial. This action is exactly what the local RF captures for us, through a local version of the Callan-Symanzik equation.

\section{The Renormalization Flow}

As far as string theory is concerned, we are interested in the spacetime action for the "couplings" of the local Hamiltonian on the worldsheet; this is the "effective" Hamiltonian attached to the entire surface, or the logarithm of the partition function. Put differently, we regard the surface as an "auxiliary" construct, defined at a length scale which we may define as 1 . Integrated amplitudes describe the effective action defined at length 1, i.e., the effective action we would attach to the surface as a whole. These amplitudes only care about the conformal structure of the surface, and not about the bare theory on the surface, defined with cutoff, in the sense that any (bare action, cutoff) pair that gives the same integrated string amplitudes is identified, in the sense of string theory and the renormalization flow. This is analogous, for example, to the considerations of [8], except that we study the flow of couplings under local rescalings, a modification we provided physical justification for in Sect. 1, where this local RF was also the motivation for (1.7).

We now want to implement the renormalization flow in the framework of Connes' geometry. Recall that in Sect. 2, we defined the notion of a lift of a connection defined on the base ring to a module over the base ring. We also defined a curvature associated to this lift. There is a natural connection on $R$ :

$$
\delta: R \rightarrow R \otimes l^{*},
$$

where $l^{*}$ is the dual of the Lie algebra, $l$, of infinitesimal conformal diffeomorphisms of $O$ and Weyl rescalings of metrics on $O$. This is defined as (for $f \in R$ )

$$
\delta_{v} f \equiv \lim _{t \downarrow 0} \frac{1}{t}\left(\alpha_{v}^{*}(t) f-f\right),
$$

where $\alpha_{v}(t)$ is any one-parameter family of diffeomorphisms such that $\dot{\alpha}_{v}(t=0) \equiv v$, for $v \in l$, with the usual action of diffeomorphisms on functions,

$$
\alpha_{v}^{*} f(x) \equiv f\left(\alpha_{v}(x)\right), \quad \forall x \in O .
$$

Clearly, infinitesimal Weyl rescalings are in the kernel of this map, since elements of $R$ do not have any dependence on the metric. This connection induces a connection 
on Fock $R$ :

$$
\delta\left(f_{1} \otimes f_{2} \ldots \otimes f_{n}\right)=\sum_{i=1}^{n} f_{1} \otimes \ldots\left(\delta f_{i}\right) \otimes \ldots f_{n} .
$$

(We do not concern ourselves with deformations of moduli in the present paper; such an extension of our approach is essential for string theory [19].)

We consider now that we are given a connection, $\nabla$, on $S$, in the sense of (2.3), that extends the connection on Fock $R$. We assume that the extension is such that

$$
\nabla: S_{n} \rightarrow\left(S_{n} \oplus S_{n+1}\right) \otimes l^{*},
$$

with the property that, for $f \in R^{\otimes n}, s \in S_{n}$,

$$
\nabla(f s)=\delta f s+(f \otimes 1) \nabla s
$$

if $\nabla s \in S_{n+1} \otimes l^{*}$; if $\nabla s=s_{0}+s_{1}$, with $s_{i} \in S_{n+i} \otimes l^{*}, i=0,1$, we set $(f \otimes 1) s_{0} \equiv f s_{0}$. To see that this makes sense, for $f, g \in R^{\otimes n}, s \in S_{n}$, we have

$$
\begin{aligned}
\nabla(f g s) & =\delta(f g) s+\{(f g) \otimes 1\} \nabla s \\
& =\{\delta f g+f \delta g\} s+\{(f g) \otimes 1\} \nabla s,
\end{aligned}
$$

and, consistent with this, we have

$$
\begin{aligned}
\nabla(f g s) & =\delta f(g s)+(f \otimes 1) \nabla(g s) \\
& =\delta f g s+(f \otimes 1) \delta g s+(f \otimes 1)(g \otimes 1) \nabla s \\
& =\{\delta f g+f \delta g\} s+\{(f g) \otimes 1\} \nabla s .
\end{aligned}
$$

Weyl rescalings of the metric are in the kernal of $\delta$. We assume that $\nabla$, restricted to the abelian subalgebra consisting of only Weyl rescalings, is compatible with the action of infinitesimal conformal diffeomorphisms on metrics (in a sense made precise in the appendix.) We call $\nabla$ the local Callan-Symanzik (CS) connection, for reasons that should be clear.

What do we demand of this connection? The partition function must be covariantly constant. This requirement is ignored (intentionally, and with good reason) in the usual treatments of the renormalizaton flow.

This connection, $\nabla$, cannot be assumed to satisfy the Leibniz rule for products in $S$; this is prevented by the operator product expansion (which we discuss below). We now wish to argue that the CS connection must be flat. If it were not flat, the local CS equations would not be integrable and the local RF would not exist. However, this implies that the global RF would not exist, since, as we argued in Sect. 1, locality and the global RF imply the local RF. The existence of the global RF is central to most of our understanding of critical phenomena and renormalization; therefore, the local CS connection must be integrable, in other words, its curvature must vanish.

One of the main reasons for the introduction of cutoffs in the study of local systems is the fact that correlations of observables are singular when we let the positions of the observables approach a common point. The operator product expansion (o.p.e.) $[31-35,16]$ studies these singularities, which are of great importance since their form determines the RF[7]. The usual form in which the 
o.p.e. is written,

$$
\phi_{i}\left(x_{1}\right) \phi_{j}\left(x_{2}{\stackrel{x_{1} \rightarrow x_{2}}{\sim}}_{\sum_{k}} c_{i j}{ }^{k}\left(x_{1}-x_{2}\right) \phi_{k}\left(\frac{x_{1}+x_{2}}{2}\right),\right.
$$

(assumed valid within arbitrary correlation functions as $x_{1} \rightarrow x_{2}$ ) does not make a great deal of sense in terms of geometry.

The fact that we deal with cutoff states implies that it is not physically correct to assume that we can localize the o.p.e. as (4.2) would have us do; since we started with smeared operators, our framework gives us a natural description of this physical data, and we now make precise what is meant by an operator product expansion in our formulation of the RF. The basic idea is that we are studying functions defined on some number of copies of $O$, and these correlation functions are singular along (some of) the diagonals of this product space. We make sense out of these singularities by introducing a cutoff, which implies that we multiply these correlation functions by cutoff functions (denoted $\chi$ in the following discussion) that vanish rapidly along diagonals. When we make a change in the cutoff function, by changing the metric by a Weyl rescaling or by a conformal diffeomorphism, the changes in a given correlation function can be represented as an integral over an additional copy of $O$, along with a smeared kernel function.

Concretely, suppose that $\rho$ is a state, with associated metric $\gamma$, and cutoff $\varepsilon$. We think of correlations in the state $\rho$, as expectations in a trace $\operatorname{Tr}[\exp (-H)$. $]$, where $H$ is the Hamiltonian associated with $\rho$. In particular, let us consider

$$
s[\gamma] \equiv \int_{U\left(x_{1}\right)} d^{2} x_{2} \rho\left(\phi_{1}\left(x_{1}\right) \phi_{2}\left(x_{2}\right) \ldots\right)
$$

is some correlation function, with $U\left(x_{1}\right)$ a small neighbourhood of $x_{1}$, and $x_{2} \in U\left(x_{1}\right)$. For simplicity, we assume that the other observables (denoted by ... in (4.3)) are at a distance larger than the cutoff, $\varepsilon$, from the boundary of $U\left(x_{1}\right)$, as mesured with the metric $\gamma$. (We are suppressing the factor of $\sqrt{ } \gamma$ in integrals, and only write $s \equiv s[\gamma]$ when we need to keep the dependence on the metric in mind.) If $w$ is an infinitesimal local Weyl rescaling of the metric $\gamma \rightarrow \exp (w) \gamma$, with $w=0$ outside $U\left(x_{1}\right)$, we expect to write $\nabla_{w} s$ as:

$$
\begin{aligned}
\nabla_{w} s \approx & \left.\int_{U\left(x_{1}\right)} d^{2} x_{0} w\left(x_{0}\right) \frac{\delta}{\delta \sigma\left(x_{0}\right)} s[\exp (\sigma) \gamma]\right|_{\sigma=0} \\
= & s \otimes s_{\beta(w)}+\text { terms due to scaling each operator } \\
& +\int_{U\left(x_{1}\right)} d^{2} x_{0} \sum_{j} c_{12}{ }^{j}\left(x_{1}, x_{2}, x_{0}\right) \rho\left(\phi_{j}\left(x_{0}\right) \ldots\right) \\
& \times\left. w\left(x_{0}\right) \frac{\delta}{\delta \sigma\left(x_{0}\right)} \chi\left(x_{1}, x_{2}\right)[\exp (\sigma) \gamma, \varepsilon]\right|_{\sigma=0} .
\end{aligned}
$$

While this form appears to be complicated, the essential features are simple to identify:

i. $\beta(w)$ (the $\beta$ operator) is the change in the Hamiltonian, $H$, associated with $\rho$, and by locality we expect it to be the integral of some local observable in a 
neighbourhood of $U\left(x_{1}\right)$. The correlation $s_{\beta(w)} \otimes s$ also incorporates a cutoff, "linking" the integral that defines $\beta(w)$ (as we just mentioned) and the integral in (4.3).

ii. $\chi$ (as mentioned above) is a cutoff function that has a functional dependence on the metric and on the cutoff $\varepsilon$ (Sect. 3) associated with the state $\rho$.

iii. The scaling terms are simple and we do not discuss them further here. They can be written in terms of a functional derivative and an added integral.

iv. The kernel $c_{i j}{ }^{k}$ makes sense in geometric terms.

v. This kernel form extends trivially to the simultaneous operator product of more than two operators.

vi. It is clear from the form of the individual terms in (4.4) that $\nabla: S_{n} \rightarrow$ $\left(S_{n} \oplus S_{n+1}\right) \otimes l^{*}$.

Computing the commutator of the RF transformations corresponding to two distinct Weyl rescalings, we obtain relations between the various terms that arise, by demanding that the curvature of the CS connection vanish, i.e., the RF exist.

\section{Physics}

We now provide a precise construction of the space of states, $\hat{A}$, using only the ring of correlation functions, $S$. In fact, we need one additional piece of data, namely, that there actually exists a state corresponding to $S$. To be precise, given the Fock $R$ module, we think of $\hat{A}$ as being a set of evaluation maps, which take an element of $S$ and assign to it the value of that correlation in a particular state.

The intuition for our definition is extremely simple: operator insertions on the string worldsheet arise as derivatives of the Hamiltonian:

$$
\operatorname{Tr}[\exp (-H+\delta H)] \approx \operatorname{Tr}[\exp (-H)]+\operatorname{Tr}[\exp (-H) \delta H]
$$

This is a standard expansion in the study of nonlinear sigma models for the purposes of string theory, corresponding to the insertion of vertex operators on the string worldsheet, (see, for example, the discussion in [7],) and is illustrated in (3.6). In our formalism, products in $S$ are insertions of additional operators on the worldsheet. We therefore consider the linear maps from $S \rightarrow S$, that are defined as multiplying correlation functions with additional operator insertions on the worldsheet. The derivative of $Z$ in the direction of an RF trajectory close to the trajectory on which $\rho_{0}$ lies, may then be written as $s_{\phi}$, where $s_{\phi} \in S_{1}$, is a one-point function. We have

$$
Z\left(\rho_{0}+\tilde{\varepsilon} \phi\right)=Z\left(\rho_{0}\right)+\tilde{\varepsilon} s_{\phi}=\left(1+\tilde{\varepsilon} s_{\phi} \otimes\right) Z\left(\rho_{0}\right),
$$

for small enough $\tilde{\varepsilon}$.

Given this intuition, we generalize it in the following way: we give ourselves an evaluation map, i.e., a state, which we call $\rho_{0}$, and we let $\operatorname{End}_{0}(S)$ be a set of endomorphisms of $S$, and $\mathrm{Aut}_{F}(S)$ denote the automorphisms (up to "finite rank") of $S$. We then define $\hat{A}\left(\rho_{0}\right)$ as follows: let $P_{0}$ be the set of paths $I \equiv[0,1] \rightarrow \operatorname{End}_{0}(S)$, and $\hat{P}_{0}$ be paths $u: I \rightarrow \operatorname{Aut}_{F}(S)$ obtained as solutions of

$$
\frac{d\left(u_{E}(t) s\left(\rho_{0}\right)\right)}{d t}=-E(t) u_{E}(t) s\left(\rho_{0}\right), \quad \forall t \in I
$$


for some $E \in P_{0}$, with the additional restriction that we have $u_{E}(0) \equiv 1$. We have the natural definition that the space of Hamiltonians is the set of automorphisms that are the endpoints of paths in $\hat{P}_{0}$ obtained as a result of solving (5.2), i.e.,

$$
\hat{A}\left(\rho_{0}\right) \equiv\left\{u \in \operatorname{Aut}_{F}(S) \mid \text { there exist } v \in \hat{P}_{0}: u=v(1)\right\} .
$$

Note that the tangent space of $\operatorname{Aut}_{F}(S)$ at a given point is identified with $\operatorname{End}_{0}(S)$. While $\hat{A}\left(\rho_{0}\right)$ might appear to be contractible, we should keep in mind that the automorphism group of a structure like $S$ is rather big, and questions of topology need to be carefully addressed. In particular, it is probably more reasonable to define the space of states as the closure of $\hat{A}\left(\rho_{0}\right)$, in $\operatorname{Aut}_{F}(S)$, in a suitable topology on $\operatorname{Aut}_{F}(S)$.

A seemingly troublesome feature in this construction is the dependence on $\rho_{0}$. This is identical to usual $\phi^{4}$ quantum field theory, for example, where we start with the action defined for fluctuations about some field configuration, usually $\phi=0$. We note that there is a natural isomorphism between the spaces $\hat{A}(\rho)$, that we determine using (5.3) for different initial states, $\rho$, by virtue of these sets being subsets of $\operatorname{Aut}_{F}(S)$. Furthermore, to be precise, we have only defined a neighbourhood of $\rho_{0}$.

We recall (1.7), which constituted our motivation for the study of the $\mathrm{RF}$ on $\hat{A}$. We are interested in extracting a space of effective Hamiltonians (equivalently, RF trajectories) from the space of Hamiltonians, by "factoring" out equivalence under the RF. The definition of $\hat{A}$ that we have just given is particularly well-suited to the study of this equivalence, as we now show. However, we must first specify the transport of the CS connection, $\nabla$ : we do this via

$$
\frac{d \nabla_{v}(t)}{d t}=\left[\nabla_{v}(t), E(t)\right], \quad \forall v \in l,
$$

$l$ being the Lie algebra of Weyl rescalings and infinitesimal conformal diffeomorphisms. This defines

$$
\nabla_{l}(\rho) \equiv\left\{\nabla_{v}(1) \text { for } v \in l\right\},
$$

as a subspace of $\operatorname{End}_{0}(S)$, at $\rho=u_{E}(1) \in \widehat{A}$, where $u_{E}(1)$ arises from $E$ via (5.2). This preserves the zero-curvature condition. The intuition here is partly derived from the notion of conjugate flows, as used in the study of dynamical systems.

We have now defined a space of states, $\hat{A}$, a natural tangent space to the space of states, $\operatorname{End}_{0}(S)$, and, at each point $\rho \in \hat{A}$, we have defined a subspace of $\operatorname{End}_{0}(S), \nabla_{l}(\rho)$. Since the curvature of the CS connection is assumed to vanish, we have a map of $l$ into the "tangent space" of $\hat{A}$, which defines an integrable subbundle of the tangent bundle of $\hat{A}$, which at each point in $\hat{A}$, has fibre $\nabla_{l}(\rho)$, as defined in (5.5). In our context, we take this to be the equivalent of a foliation of the space of field theories $\hat{A}$.

Spaces of leaves of foliations (here, the space of $\mathrm{RF}$ trajectories) are topologically rather ill-behaved. As mentioned above, one of Connes' main motivations was to attach meaningful algebras to such degenerate spaces. In a straightforward analogy with the notation in Sect. 2, we form the holonomy groupoid, which we call the renormalization groupoid, with the data described in the previous paragraph, and denote it $\left(\hat{A}, \nabla_{l}\right)$. As far as we are concerned, $S$ is the fundamental object and $\hat{A}$ is a 
derived object. Explicitly, we consider paths in $\hat{A}$ whose tangents at each point on the path lie in the subspace of the tangent space to $\widehat{A}$, defined via (5.4). $\rho_{i} \equiv u_{i} \in \hat{A}$, $i=1,2$, are on the same RF trajectory if there exists $E \in P_{0}$ with associated $u_{E}(t) \in \operatorname{Aut}_{F}(S)$, such that $u_{E}$ solves (5.2) with the boundary conditions: $u_{E}(0)=u_{1}, u_{E}(1)=u_{2}$. The holonomy is computed for these paths via the Bott connection (Sect.2), (or the GNS connection [22]) with the map $q$ : $\operatorname{End}_{0}(S) \rightarrow$ $\operatorname{End}_{0}(S) / \nabla_{l}$, and this completes the definition of the renormalization groupoid. It will be noted that for every leaf, this holonomy is a measure of the "twisting" of physical correlations (i.e., those that are annihilated by the local CS connection) as we move about on a particular RF trajectory. These are the integrated string amplitudes we assign to this RF trajectory.

We have now assembled the basic structure for understanding the RF equivalence structure on the space of Hamiltonians. We must consider measure theory on renormalization groupoids so as to make sense of the decomposition in (1.7), since we expect the string action to be a transverse measure on the renormalization groupoid.

There are some questions involved as to what we should allow in $\operatorname{End}_{0}(S)$. The most natural definition, given that we based our construction on locality in the RF, appears to include multiplication by any linear combination of elements in $S_{1}$, which would include operators smeared over only part of the worldsheet. Furthermore, the work of Fischler and Susskind [37] provides us with very interesting "topological" operators in $\operatorname{End}_{0}(S)$. Therefore, in this paper, we assume that End $(S)$ is generated by the image of $l$ under $\nabla$, by the above-mentioned "multiplication" (by elements in $S_{1}$ ) endomorphisms, and by "field redefinitions" (which we consider below). More precisely, we assume that End $(S)$ is generated (as a ring) by a set of endomorphisms, $\operatorname{End}_{0}(S)$, with all elements $E \in \operatorname{End}_{0}(S)$ satisfying $E: S_{n} \rightarrow S_{n} \oplus S_{n+1}$.

The automorphism group that one associates to the CS connection is the "gauge group" of our construction. This trivially includes the transport of the RF from one point of an RF trajectory to another. Another part of it arises from "field redefinitions," i.e., automorphisms of the algebra $A$. We note that endomorphisms of $A$ have the obvious lift to Fock $A$, and they then lift to $S$, canonically. These are the infinitesimal classical field redefinitions, which we denote as $\operatorname{End}(A, S) \subset \operatorname{End}(S)$. We can consider which of these field redefinitions continues as a symmetry in the quantum theory: a minimal requirement is that the quantum mechanical symmetries of the theory are those elements of End $(A, S)$ which commute with the local RF endomorphisms, $\nabla_{v}$. We can extend the foliation to include directions generated by these "tangent vectors," or we may view these as additional symmetries of the space of leaves of the renormalization flow that we have considered so far. The latter point of view is preferred as far as (1.7) is concerned. Another part of the automorphism group of the flat CS connection is a suitable set of deformations of the cutoff function $\chi$ in (4.4). This is one sense in which the CS connection depends on the particular (physical) regularization that we picked.

Given the dynamical systems analogies that appear naturally in our formalism, we turn now to another aspect of the renormalization flow with some dynamical systems connections, namely, we consider the entropy of the RF. We define the relative entropy ([38] and references therein) between two states, $\rho_{i}, i=1,2$, which 
we naïvely identify with traces corresponding to two Hamiltonians, $H_{i}, i=1,2$, as

$$
\mathscr{S}\left(\rho_{1}, \rho_{2}\right) \equiv \mathscr{S}\left(H_{1}, H_{2}\right) \equiv \operatorname{Tr}\left[\exp \left(-H_{1}\right)\left(H_{1}-H_{2}\right)\right] .
$$

With a local choice of coordinates on $\hat{A}$, around $H_{1}$, we define an analogous differential construct,

$$
d \mathscr{S}\left(H_{1}\right) \equiv \operatorname{Tr}\left[\exp \left(-H_{1}\right) d H_{1}\right],
$$

which is a "1-form" on $\hat{A}, d \mathscr{S}\left(H_{1}\right) \in \operatorname{End}_{0}(S)_{H_{1}}^{*}=\operatorname{End}_{0}(S)^{*}$; this 1 -form maps a "vector" $a \in \operatorname{End}_{0}(S)$ to $d \mathscr{S}(a) \equiv a Z \in S$. Note that $\nabla_{l}\left(H_{1}\right)$ is in the kernel of $d \mathscr{P}$, since, by definition, the partition function is constant along RF trajectories. This may be construed as the statement that the one-point function of the $\beta$ operator is 0 , where we think of $\beta \equiv \sum \beta^{i} \phi_{i}$, for $\phi_{i} \in A$, in the sense of (3.6) and (4.4) [7]; we think of $d \mathscr{S}(\beta)\left(H_{1}\right) \equiv s_{\beta}\left(H_{1}\right)$. (Of course, we determine a different $\beta$ for every element of $l$, so we might think of $\beta$ more abstractly as an element of $\operatorname{End}_{0}(S) \otimes l^{*}$; this is natural in terms of our definition of $\nabla$.)

We note another connection of beta functions with this relative entropy: if $\rho_{i}$, $i=0,1,2$, are states corresponding to Hamiltonians $H_{i}, H_{0}=H_{1}+\varepsilon \phi$, some $\phi \in A$, and $H_{2}$ is obtained from $H_{1}$ by an infinitesimal RF transformation, $H_{2}=$ $H_{1}+\tilde{\varepsilon} \beta(v), v \in l$, then

$$
\mathscr{S}\left(H_{2}, H_{0}\right)-\mathscr{S}\left(H_{1}, H_{0}\right)=\operatorname{Tr}\left[\exp \left(-H_{1}\right)(\varepsilon \tilde{\varepsilon} \beta(v) \phi)\right] \equiv \varepsilon \tilde{\varepsilon}\left[s_{\beta(v)} \otimes s_{\phi}\right]\left(H_{1}\right) .
$$

By expanding $\beta(v)$ as in the previous paragraph, and making use of the GNS Hilbert space inner product, it is possible to extract the values of each beta function separately. These beta functions are therefore seen to be closely connected with infinitesimal changes in relative entropy.

We now turn to a discussion of Zamolodchikov's seminal observation [39] regarding the entropy of the renormalization flow. (The first part of) Zamolodchikov's $c$-theorem states that there is a function defined on the space of couplings which decreases along RF trajectories. We provide a simple argument that is a mild generalization of Zamolodchikov's result. Consider a naïve decomposition of the space of Hamiltonians into a space of couplings and a space of metrics. We will show that there is a function on the (sub)space of renormalizable couplings that decreases in the direction of the RF flow, for constant Weyl rescalings. Denote $\beta(V)$ as "the" beta operator, namely, the beta operator corresponding to a global increase in the cutoff. Then, we consider $\left.\mathscr{C} \in \operatorname{End}_{0}(S)^{*}\right|_{H}$, such that for $\left.t \in \operatorname{End}(S)_{0}\right|_{H}, t: \nabla^{A} t=0$, i.e., operators of canonical dimension $2,\left.\mathscr{C}(t)\right|_{H}=$ $-S_{\beta(V) \otimes t}(H)$. For these renormalizable directions in the tangent space to the space of couplings, we have

$$
\left.\mathscr{C}(\beta(V))\right|_{H}=-s_{\beta(V) \otimes \beta(V)}(H) .
$$

If the GNS metric had positive signature, this would imply Zamolodchikov's result, provided that $\mathscr{C}$ is a closed form. Let us compute $d \mathscr{C}$ on the subspace of renormalizable couplings:

$$
\begin{aligned}
d \mathscr{C}(t, x) & =s_{t} \otimes \nabla_{V} s_{x}+\left[\nabla_{V}, s_{t} \otimes\right] s_{x}-(t \leftrightarrow x) \\
& =\nabla_{V}\left(s_{t} \otimes s_{x}\right)-\nabla_{V}\left(s_{x} \otimes s_{t}\right)=0, \quad t, x \in A, \quad \text { with } \quad \nabla^{A} x=\nabla^{A} t=0 .
\end{aligned}
$$


We have used (5.4) here, and the fact that $s_{t} \otimes s_{x}=s_{x} \otimes s_{t}$. The space of couplings is simply connected, so closed forms are exact, and, therefore, $\mathscr{C}$ is the differential of some function, which we identify with Zamolodchikov's $c$-function. By (5.6), the $c$-function decreases along renormalization trajectories. In fact, it decreases in any direction such that $s_{\beta(w) \otimes \beta(V)}>0, w \in l$; this leads us to conclude that this decrease holds for a small cone about $V$ in $l$. It goes without saying that our argument is not as physically compelling as Zamolodchikov's; it may have some merit in a more general approach.

\section{To Conclude}

To conclude: we have formulated a local renormalization flow, on spaces of twodimensional field theories. This two-dimensional local renormalization equivalence is a physically natural candidate for the gauge symmetry of string theory. Analogies with statistical physics were important in all aspects of our discussion. In a sense, we have formulated string theory without strings. As a consequence, our formulation makes no reference to string perturbation theory. The physics of string theory lies in transverse measures on the space of local RF trajectories. There is no natural way to separate the dynamics from the kinematics in our formulation. We gave a physical argument for why critical models are important for these measures.

In more detail, we used some of Connes' geometric constructs to define a renormalization groupoid as a quotient of a space of two-dimensional field theories by the local renormalization flow, via a local CS connection. A minimal definition of the data we require to define a renormalization groupoid is:

1. a module, $S$, over Fock $R$, with a graded ring structure, and identity, $Z$,

2. a flat connection, $\nabla: S \rightarrow S \otimes l^{*}$, satisfying $\nabla\left(S_{n}\right) \subset\left(S_{n} \oplus S_{n+1}\right) \otimes l^{*}$, extending (in the sense of Sects. 2 and 3) the action of $l$ on $R$, such that $\nabla Z \equiv 0$, and

3. a set of endomorphisms of $S, \operatorname{End}_{0}(S)$, such that elements in $\operatorname{End}_{0}(S)$ map $S_{n} \rightarrow S_{n} \oplus S_{n+1}$.

We seek invariants of such renormalization groupoids, to classify them; these invariants would be analogous to the dimension of spacetime in general relativity. Our construction only gives us a path-connected component of the space of field theories we would like to attach to a given set of observables, though the closure may be topologically non-trivial.

An important observation is that renormalization groupoids are local on spaces of field theories, i.e., can be constructed for a small neighbourhood of a given twodimensional field theory. Local calculations of the renormalization flow are important ([40] and references therein).

We note connections of our work with previous work in related areas. The idea that different cutoffs are merely different descriptions of the same effective physics is very old. It is implicit in the earliest works on the renormalization of quantum field theories, and has certainly been quite explicit since the appearance of the first papers on the renormalization flow $[41,42]$. The geometric local formulation of this gauge invariance that we have presented is clearly inspired by the symmetries of string theory and the suspected relation (see, e.g., [36]) between 
string theory equations of motion and beta functions of the renormalization flow [6]. Wilson [3] discusses the analogy between the derivative and the renormalization flow; the CS connection is a formulation of renormalization transformations as (generalized) derivatives.

The idea that spaces of two-dimensional field theories are possible candidates for configuration spaces of string theory is not new (see, e.g., [13]). Banks and Martinec [14] were the first to take it seriously. They considered a possible description of the renormalization flow in the neighbourhood of fixed points and discussed the possibility of deriving the beta function from an "actional" on a space of twodimensional field theories. They noted that it should be possible to calculate tree-level string scattering amplitudes from the beta functions. Since we expect to be able to calculate tree-level scattering amplitudes from the equations of motion of a given field theory, this is equivalent to the belief that beta functions are the classical equations of motion of string theory. They indicate that future work will develop their formalism further. It would be very interesting to understand the connections between their ideas and the framework that we have presented here.

Every construction in our work was genus-independent. We shall extend our constructions to include deformations to moduli. This involves extending our construction to the same construction defined over a base space, in the sense of algebraic geometry. Hence, we consider a sheaf, $\mathscr{R}$, over a topological space, $\mathscr{N}$, and a map $p: \mathscr{R} \rightarrow \mathscr{N}$, such that the inverse image of every point in $\mathscr{N}$ is a function ring of the form Fock $R$. Keeping Fock $A$ fixed, we define all the rest of our constructions over every point in $\mathscr{N}$ exactly as above, and then require that all dependence on $\mathscr{N}$ be continuous. This is essential to a natural understanding of the problem.

Where does the perturbation theory embodied in (1.4) fit into this picture? We have not developed the necessary tools to answer this question, in the sense that we do not, as yet, have a concrete understanding of the rôle of the base space, $\mathscr{N}$. Before we attempt to extract (1.4) from our formalism, we must consider more tractable problems, such as doing explicit calculations of renormalization flows on spaces of field theories defined on, say, a sphere. This is much better defined in terms of explicit computations, and this work is currently in progress [19]. It is straightforward to show that the Virasoro-Shapiro amplitude is obtained by looking for invariants of local rescaling for bosonic free fields on a sphere $[19,22]$.

We have omitted several topics of interest for both string theory and statistical mechanics. Introducing fermions into $A$ is a triviality. The question of spin structures and superselection sectors in the space of states, or in the ring of correlation functions, is more subtle, but can be understood naturally in this formalism. While we have not discussed other applications at any length in the above, it is of great interest to consider applying this formalism to gauge theories, where we could, for example, consider the action of gauge transformations and the local RF on spaces of cutoff, gauge-fixed Hamiltonians, described by means of a ring of correlations.

\section{Appendix}

$\operatorname{Hom}(X, Y)$ will refer to the set of morphisms from $X$ to $Y$. When $Y=X$ is an 
abelian group, this set is a ring, which we denote End $(X)$. The endomorphisms of a module (ring) will always refer to homomorphisms from the additive group of the module (ring) into itself. A module, $S$, over a ring, $R$, is an additive group with a ring homomorphism from $R$ to $\operatorname{End}(S)$. $\operatorname{End}_{R}(S)$ denotes the subring of End $(S)$ that consists of elements that commute with all elements in the image of $R$ in $\operatorname{End}(S)$.

Since we only used the concept of a sheaf in connection with vector bundles, we give only a restricted definition. Suppose $X$ is a smooth manifold and $E$ is a vector bundle over $X$, with projection map $\pi$. Consider an open set $Y \subset X$, and a smooth map $s_{Y}: Y \rightarrow E_{Y}$, where $E_{Y}$ denotes the restriction of the vector bundle to $Y$. Such a map is called a section of $E_{Y}$ if $\pi{ }^{\circ} S_{Y}=1_{Y}$, the identity map on $Y$. The set of all sections of $E_{Y}$ over a given open $Y \subset X$ is an abelian group, since the fibres of $E$ are vector spaces. The sheaf $\Gamma(E)$ is the map from the set of open sets of $X$ to the category of abelian groups, which assigns to each open set, the abelian group of sections of $E$ above that open set. We will not describe the topology of the sheaf, since we do not use it in this work.

Let $V$ and $W$ be smooth manifolds, and $f: V \rightarrow W$ a smooth map. We have the induced map, called the differential of $f$, mapping the tangent bundle of $V$ to the tangent bundle of $W$,

$$
\begin{aligned}
T_{*} f: T V & \rightarrow T W \\
\left(x, v_{x}\right) & \mapsto\left(f(x),\left[T_{*} f_{x}\right] v_{x}\right),
\end{aligned}
$$

where $T_{*} f_{x}$ is a linear map from $T V_{x} \rightarrow T W_{f(x)}$. $f$ is called a submersion when $T_{*} f_{x}$ is surjective for every $x \in V$. A smooth sub-bundle, $F$, of the tangent bundle of a manifold is said to be integrable, or a foliation of $V$, if it is locally the kernel of the differential of a submersion, $F \equiv \operatorname{ker}\left(T_{*} f\right)$. The geometric picture is simple: if $V(W)$ is a manifold of dimension $m(n)$, with $m>n$, then if $f$ is (locally) a submersion, we have a (local) decomposition of $V$,

$$
U=\bigcup_{y \in W} f^{-1}(y) \cap U
$$

where $U$ is a small enough open subset in $V$. Sets of the form $f^{-1}(y)$ are called the leaves of the foliation.

For a given differential manifold, the space of Riemannian metrics is fibered by the relation of conformal equivalence. Weyl rescalings of the metric correspond to motion along the fibres. There is also a natural action of diffeomorphisms of the manifold on this space of metrics. Denote the Lie algebra of Weyl rescalings by $\mathscr{W}$, the tangent space of the space of Riemannian metrics by $T$, and the Lie algebra of infinitesimal conformal diffeomorphisms by $C$. The connection, $\nabla$, is assumed compatible with this fibration, i.e., $\nabla \equiv \nabla^{(1)}+\nabla^{(2)}$, with $\nabla^{(1)}$ a connection on $l$ that factors through $\Gamma(T)$ in the sense that if $w \in \mathscr{W}$ and $c \in C$ are such that their images in $\Gamma(T)$ (under the obvious maps) are equal, $\nabla_{w}^{(1)}=\nabla_{c}^{(1)}$. Furthermore, we require that $\nabla_{\mathscr{W}}^{(2)}=0$. 
this manuscript. I have benefited from conversations with D. Brydges and T. Spencer, B. Ratra (who pointed out the existence of [7]), A. Wightman, L. Yaffe, and T. Banks.

\section{References}

1. Haag, R., Kastler, D.: An algebraic approach to quantum field theory. J. Math. Phys. 5, 848-861 (1964)

2. Wilson, K. G., Kogut, J. B.: The renormalization group and the $\varepsilon$ expansion. Phys. Rep. 12C, 75-199 (1974)

3. Wilson, K. G.: The renormalization group: Critical phenomena and the Kondo problem. Rev. Mod. Phys. 47, 773-840 (1975)

4. Kadanoff, L. P.: Scaling, Universality and operator algebras. Phase transitions and critical phenomena, Domb, C., Green, M. S., (eds.), v. 5a, 1-34, New York: Academic Press 1976

5. Polyakov, A. M.: Microscopic description of critical phenomena. Sov. Phys. JETP 28, 533-539, (1969); Properties of long and short range correlations in the critical region. 30, 151-157, (1970); NonHamiltonian approach to conformal field theory. 39, 10-18 (1974)

6. Friedan, D. H.: Nonlinear models in $2+\varepsilon$ dimensions. Ann. Phys. 163, 318-419 (1985)

7. Polyakov, A. M.: Directions in string theory. Lecture at the International Congress of Mathematicians, Berkeley (July 1986)

8. Polchinski, J.: Renormalization and effective Lagrangians. Nucl. Phys. B231, 269-295 (1984)

9. Warr, B.: Renormalization of gauge theories using effective Lagrangians. Caltech preprints CALT68-1334, 1393 (1986)

10. Phase transitions and critical phenomena. Domb, C., Green, M. S. (eds.), v. 6, New York: Academic Press 1976

11. Amit, D. J.: Field theory, the renormalization group, and critical phenomena, 2nd, ed., Singapore: World Scientific 1984

12. Patashinskiĭ, A. Z., Pokrovskiĭ, V. L.: Fluctuation theory of phase transitions. Shepherd, Tr. P. J. (ed.) Oxford: Academic Press 1979

13. Lovelace, C.: Stability of string vacua (I). A new picture of the renormalization group. Nucl. Phys. B273, 413-467 (1986)

14. Banks, T., Martinec, E. J.: Renormalization group and string field theory. Nucl. Phys. B294, 733-746 (1987)

15. Belavin, A. A., Polyakov A. M., Zamolodchikov, A. B.: Infinite conformal symmetry in twodimensional quantum field theory. Nucl. Phys. B241, 333-380 (1984); Infinite Conformal Symmetry of Critical Fluctuations in Two Dimensions. J. Stat. Phys. 34, 763-774 (1984)

16. Gross, D. J.: Applications of the renormalization group to high-energy physics. Les Houches Summer Institute 1975. Balian, R., Zinn-Justin. J., (eds.), pp. 141-250, Amsterdam: North-Holland 1976

17. Schoen, R.: Morse lectures. The Institute for Advanced Study, Princeton (November 1987)

18. Connes, A.: Sur la theorie non commutative de l'integration. Algèbres de Opérateurs. Harpe, P. de la (ed.), Lecture. Notes in Mathematics vol. 725, pp. 19-143, Berlin, Heidelberg, New York: Springer 1979

19. In preparation; to appear in Mod. Phys. Lett. A

20. Emch, G. G.: The $C^{*}$-algebraic approach to phase transitions. Phase transitions and critical phenomena, Domb, C., Green, M. S., (eds.), v. 1, 137-175, New York: Academic Press 1972

21. Thirring, W.: A course in mathematical physics, vol. 4, Berlin, Heidelberg, New York: Springer 1983

22. Periwal, V.: Princeton University dissertation (1988)

23. Connes, A.: $C^{*}$ algèbres et géométrie differentielle. C. R. Acad. Sci. Paris Ser A 290 599-604 (1980); Non-commutative Differential Geometry. Pub. Math. I.H.E.S. 62, 41-144 (1986)

24. Connes, A.: A survey of feliations and operator algebras. Proc. Symp. in Pure Math., A.M.S, 38, (Part 1) 521-628 (1982)

25. Connes, A.: Lectures at the NATO-ASI Cargèse Summer School, July 1987

26. Karoubi, M.: K-theory. Berlin, Heidelberg, New York: Springer 1978 
27. Bott, R.: On a topological obstruction to integrability. Proc. Symp. in Pure Math., A.M.S., 16, 127132 (1970); On topological obstructions to integrability, Proc. International Congress of Mathematicians (Nice, 1970). v. 1, 27-36. Paris: Gauthier-Villars 1971

28. Polchinski, J.: Vertex operators in the polyakov path integral. Nucl. Phys. B289, 465-483 (1987)

29. Wightman, A. S., Gårding, A.: Fields as operator-valued distributions in relativistic quantum theory. Arkiv. Fysik 28, 129-184 (1964)

30. Callan, C. G., Gan, Z.: Vertex operators in background fields. Nucl. Phys. B272, 647-660 (1986)

31. Ripper, J.: In: Dr. Strangelove, dir. S. Kubrick, 1962

32. Wilson, K. G.: Non-Lagrangian models of current algebras. Phys. Rev. 179, 1499-1512 (1969); Renormalization group and strong interactions. D3, 1818-1846 (1971)

33. Zimmerman, W.: Local operator products and renormalization in quantum field theory, Brandeis Summer Inst. in Theor. Phys., 1970. vol 1, pp. 395-589. Deser, S., Grisaru, M., Pendleton, H., (eds.). Cambridge: MIT Press 1970

34. Callan Jr C. G.: Introduction to renormalization theory. Les Houches Summer Institute 1975, Balian, R., Zinn-Justin J., (eds.), pp. 41-78. Amsterdam: North Holland 1976

35. Brézin, E.: Applications of the renormalization group to critical phenomena, Les Houches Summer Institute 1975, Balian, R., Zinn-Justin, J., (eds.), pp. 329-386. Amsterdam: North-Holland 1976

36. Gross, D. J.: The Heterotic String. Proc. Santa Barbara Workshop on Unified String Theories, pp. 357-399. Green, M. B., Gross, D. J., (eds.), Singapore: World Scientific, 1986, and references therein

37. Fischler, W., Susskind, L.: Dilaton tadpoles, string condensates and scale invariance. Phys. Lett 171B, 383-389 (1986), 173B, 262-266 (1986)

38. Connes, A., Narnhofer, H., Thirring, W.: Dynamical entropy of $C^{*}$ algebras and von Neumann algebras. Commun. Math. Phys. 112, 691-719 (1987)

39. Zamolodchikov, A. B.: Irreversibility of the flux of the renormalization group in a $2 \mathrm{D}$ field theory. JET Lett. 43, 730-732 (1986)

40. Klebanov, I., Susskind, L.: Renormalization group and string amplitudes. Phys. Lett. 200B, 446 (1988)

41. Stueckelberg, E. C. G., Petermann, E.: La normalization des constantes dans la theorie des quanta. Helv. Phys. Acta 26, 499-520 (1953)

42. Gell-Mann, M., Low, F. E.: Quantum electrodynamics at small distances. Phys. Rev. 95, 1300-1312 (1954)

Communicated by L. Alvarez-Gaumé

Received April 13, 1988; in revised form June 22, 1988 
